\title{
Explorative Experiments in Autonomous Robotics
}

\author{
Francesco Amigoni and Viola Schiaffonati \\ Artificial Intelligence and Robotics Laboratory, Politecnico di Milano, Italy
}

\begin{abstract}
The debate on the experimental method, its role, its limits, and its possible applications has recently gained attention in autonomous robotics. If, from the one hand, classical experimental principles, such as repeatability and reproducibility, play as an inspiration for the development of good experimental practices in this research area, from the other hand, some recent analyses have evidenced that rigorous experimental approaches are not yet full part of the research habits in this community. In this paper, in order to give reason of a part of the current experimental practice in autonomous robotics that cannot be satisfactorily accommodated under the traditional concept of controlled experiment, we will advance the notion of explorative experiment. Explorative experiments in this context should be intended as a form of investigation carried out in the absence of a proper theory or theoretical background, where the control of the experimental factors cannot be fully managed from the beginning. We show that this notion arises from (and is supported by) the analysis of the experimental activities reported in a significant sample of papers that have been given awards at two of the largest and most impacting robotics research conferences.
\end{abstract}

\section{Introduction}

The discussion on experiments and the effort in developing good experimental methodologies have gained attention in autonomous robotics in the very last years. This field is oriented to develop robot systems ${ }^{1}$ that are autonomous in the sense that they have the ability to operate without continuous human intervention, in order to work in places hardly accessible by humans or in cooperation with humans in common environments. In autonomous robotics, human operators evolve from being active controllers of the robot systems to being more passive supervisors of the same robot systems.

The increasing attention to experimental issues in this field can be attributed to many factors. For sure, disciplinary and scientific ones play an important role, as

\footnotetext{
${ }^{1}$ Generally speaking, and for the purpose of our presentation, a robot system is an artifact that interacts with the external environment through its sensors and actuators and that is controlled by software programs.
} 
autonomous robotics strives for reaching the same methodological standards of other scientific disciplines. Also practical accomplishments are essential in promoting standard ways to measure performance and parameters (e.g., those related to safety). Finally, commercial purposes are emerging with the aim of having standard benchmarks to evaluate products. Accordingly, a number of initiatives have been promoted, ranging from workshop series [Bonsignorio et al., 2015], to special issues of journals [Bonsignorio and del Pobil, 2015], to European projects funded under different programs [Rawseeds, 2015; RoCKIn, 2015], to a generalized interest to experimental issues.

When analyzing the experimental trends emerging in the community, two different tendencies can be observed: on the one hand, the principles of experimental method (such as comparison, reproducibility, repeatability, justification, and generalization) play an inspirational role in the direction of defining a more rigorous approach to experiments; on the other hand, these rigorous approaches are not yet full part of the current research practice in robotics. For instance, from the systematic analysis presented in [Amigoni et al., 2014], it emerges that only few of the experiments conducted in a significant sample of autonomous robotics articles come close to controlled experiments in the sense employed by [Tedre, 2015] for computing in general.

To better investigate the nature and the role of experiments in autonomous robotics within this heterogeneous and dynamic context, we believe that the current debate needs to be widened. It has to take into account not only the traditional tools of the philosophy of science, in the form of the philosophy of experimentation, but also other disciplines, both already existing (such as the philosophy of technology) and under development (such as the interdisciplinary field labelled philosophy and engineering). In this paper, we propose to stretch the traditional idea of experiment, with the aim of introducing the (still preliminary) notion of explorative experiment to give reason of a part of the current practice in autonomous robotics. To a first approximation, explorative experiments are forms of empirical investigation on the functioning of artifacts and on their interaction with the environment, in absence of a proper theory or theoretical background and without the typical constraints of controlled experiments. Our main original contribution is thus a step toward an enlarged framework that can satisfactorily account for all the different forms of experimentation in autonomous robotics.

With the aim of making the discussion more concrete, and without any attempt of being exhaustive, we analyze the papers that in the last years have been given awards at the IEEE International Conference on Robotics and Automation (ICRA) and at the IEEE/RSJ International Conference on Intelligent Robots and Systems (IROS), which are two large and impacting robotics research conferences. Our goal is to show how the notion of explorative experiment emerges from the current practice and how it can give reasons of some current experimental activities in autonomous robotics, in addition and beyond the traditional notion of experimentation. 
In the following, we widen the framework of experimentation by considering the many faces of experiments already proposed for computing ${ }^{2}$ and the notion of directly action-guiding experiments, and by analyzing the crisis of the traditional experimental paradigm, as it has been conceptualized for experiments with new technologies (Section 2). We, then, survey how experiments are conducted in the current practice of autonomous robotics and how they can fit within the already existing categories of experiments in computing (Section 3). Finally, we advance a definition of explorative experiments capable to take into account a significant part of the current experimental practice in autonomous robotics (Section 4).

\section{Widening the experimental framework}

In this section, we introduce the concepts that enable to enlarge the framework for reflecting on experimentation in autonomous robotics.

The many faces of experimentation. The term 'experiment' is used in the field of computing in a variety of ways. As it has been reconstructed in detail by [Tedre, 2015], at least five different views of experiments can be recognized in the practice of the field. There are the so called feasibility experiments aimed at empirically demonstrating ('demonstration' and 'experiment' are terms commonly used as synonymous in computing) the proper development and working of a technology. There are trial experiments, evaluating some aspects of a system using predetermined variables in a laboratory, and field experiments, aimed at evaluating these aspects of a system outside the laboratory, in the real world. There are also comparison experiments devoted to compare different solutions to look for the best one for a specific problem. And, finally, there are controlled experiments, those more similar to the traditional notion of experimentation and aimed at achieving generalization and prediction. What is important in this account is not how the notion of experiment should be used, but how it is actually used: "Many would object against calling, for instance, feasibility demonstrations 'experiments,' arguing that the term 'experiment' has a special meaning in science. They are right. But if one looks at how authors in computing have used the term-not how it should be used - those five uses are easily found" [Tedre, 2015, 190]. The differences introduced by these categories are surely of great importance in our discussion and in the next sections we will argue for their extension to autonomous robotics at the light of the experimental activities reported in the papers we surveyed.

${ }^{2}$ In this work we use the term "computing", "computer science", and "computer science and engineering" in an interchangeable way to name the academic discipline. While recognizing the relevant difference between the theoretical and practical ends of the computing spectrum, introducing a taxonomy is beyond our scope here. 
Directly action-guiding experiments. Besides the traditional notions of experiments, such as the ones just presented, we introduce here that of directly actionguiding experiments, as technological forms of experimentation already present in pre-scientific times. In particular, the difference between epistemic experiments and directly action-guiding experiments, as recently conceptualized in [Hansson, 2015], can help emphasizing not only that explorative experiments we discuss in this paper are performed on artifacts (and not on natural phenomena), but also that they have different purposes than the epistemic ones. An experiment is epistemic when it aims at providing information about the workings of the natural world, whereas an experiment is directly action-guiding when it satisfies two criteria: (a) the outcome looked for consists in the attainment of some desired goal of human action and (b) the interventions studied are potential candidates for being performed in a non-experimental setting in order to achieve that goal. A clinical trial of an analgesic is one of the examples provided by Hansson to illustrate a directly action-guiding experiment, where the outcome looked for is the efficient pain reduction and the experimental intervention is the treatment that might be administered. A systematic test on an autonomous robot employed to assist an elderly person in her home is also an example of a directly action-guiding experiment: the outcome looked for is the proper interaction of the robot with the person and the experimental intervention consists in the careful tuning of the abilities that the robot must possess to positively achieve this goal.

Exploratory experiments. Directly action-guiding experiments contribute also to introduce an explorative element that characterizes experimentation in autonomous robotics, as we will see in the next sections. The concept of experiment as exploration is not new. For example, in some recent philosophical research, exploratory experimentation labels those forms of experimentation in science which are not always guided by theories. One of the first authors to recognize the epistemic importance of exploratory experiments [Steinle, 1997] defines exploratory experimentation as driven by the desire to obtain empirical regularities when no well-formed theories or no conceptual frameworks are available. What is important in this characterization (that in this case is based on a detailed reconstruction of the early research in electromagnetism) is that the experimental activity may be highly systematic and driven by the typical experimental guidelines, despite its independence from specific theories. The same term is used with a slightly different meaning in another article appeared in the same year but in the context of some early research in protein synthesis [Burian, 1997], where exploratory experimentation is seen as a style of inquiry not guided by theory. These and other similar works are mainly directed to contrast the theory-driven approaches of most of the philosophy of science in the spirit of experimentation as having a life on its own [Hacking, 1983]. Even if they recognize that exploratory experimentation is typically not completely free of theory, they aim at showing that the epistemic significance of those inquiries are not primarily theory-driven by presenting several detailed case studies. The idea that "the aim of exploratory experiments is to generate significant findings about phenomena without appealing to a theory about these phenomena for the purpose of focusing experimental attention on a limited 
range of possible findings" [Waters, 2007, 5] is probably that serving better as an inspiration for more recent works devoted to provide evidence of the exploratory shift observed in the methodology of some areas of biology [Franklin, 2005].

Experimental control. Controlling the experimental factors that are investigated constitutes one of the key issues of the experimental method. To deploy an experimental system, knowledge and control of the interactions between the system and its environment need to be managed. Controlled experiments are usually performed having in mind quite precise expectations of the possible outcomes. The research questions are clearly stated and the hypotheses to be investigated are made explicit. Then, on a general account, experiments are designed and performed varying the different experimental parameters in order to determine which of the different experimental conditions are indispensable and, then, looking for stable empirical rules. For producing stable and repeatable experiments, experimenters vary a number of factors in their experimental systems to examine whether they are relevant or not. The fact that experiments are performed in laboratories responds exactly to this attempt of control.

The crisis of the traditional notion of control. Traditionally the control paradigm for experimentation, as it has been devised in the history of science, relies on two assumptions [Kroes, 2015]: the experimenter is not part of the system on which the experiment is performed and (s)he is in control of the independent variables and of the experimental set-up. Accordingly, the experimenter is able to intervene both by changing these variables to evaluate their influence on the dependent ones and by varying the experimental set-up. This traditional control paradigm becomes problematic, and a consequent shift in the notions of intervention and control is observed, when considering new technologies as sociotechnical systems, namely as hybrid systems composed of natural objects, technical artifacts, human actors, and social entities. The idea of controlling the experimental system from a center of command and control that is outside the system becomes highly problematic [Kroes, 2015]. Reasons are that the distinction between the experimental system and its environment is critical, but also that the environment is complex, where complexity arises from the co-presence of technical artifacts and natural and social elements.

It is interesting to note that the same crisis in the traditional notion of control can be observed also in a part of the current experimental practice in autonomous robotics. Although the kind of technology we are discussing here does not possess in a full and complete way the features of large-scale socio-technical systems, such as the world civil aviation system [Vermaas et al., 2011], it nevertheless shares some of their characteristics. We could say that the experimental system in the case, for instance, of experiments with autonomous robots is hybrid, in the sense that not just technical components play an essential role for the functioning of the system, and thus have to be evaluated, but also natural objects, human actors, and social entities need to be taken into account (e.g., for their interaction with the robot systems). Moreover, if in the natural sciences it is prescribed that the experimenter should be an outsider of the phenomenon to be investigated, it is not clear how a person developing autonomous robots, namely computation-based 
artifacts, could be an outsider with respect to a phenomenon (i.e., an artifact) that (s)he has created [Tedre, 2011]. Except for some significant examples, in autonomous robotics, tests on the artifacts are usually performed by the same people that created them, losing the sort of independence of the experimenter that is prescribed in the classical experimental protocol.

One could ask what is the reason for a robotician to test the artifacts that (s)he has developed and, thus, should know in detail. To answer this question, it is important to recognize at least two sources of unpredictability, arising in the artifact, due to its complex nature, and in its interaction with the physical environment (including humans) surrounding it. This is particularly evident in the case of autonomous robotics, where the goal is that of having robots that do not require continuous human supervision. Autonomous robots are very complex entities composed of interacting modules ranging from sensors, to actuators, to software programs, whose overall behavior is hardly predictable, even by their own designers, especially when considering their interaction with the external physical (and social) world. Not only tests that a given robot is working properly (and possibly better than others) have to be performed without the required independence of the experimenter, but also autonomous robots have to be tested for their proper interaction with environments (including in most of the cases other human beings) that is hardly predictable.

In summary, autonomous robotics, as several other new technologies, can benefit from a wider framework in which its experimental activities can be discussed, as we further argue in the following of this paper.

\section{A survey of different experiments in autonomous robotics}

In this section, we present some considerations emerging from the survey we have conducted on the papers that have been awarded the Cognitive Robotics Best Paper Award and the CoTeSys (Cognition for Technical Systems) Cognitive Robotics Best Paper Award at ICRA and at IROS, respectively, from 2010 to 2015. In total, we consider 11 papers that we deem represent a significant sample of current research on autonomous robots, as they are witnessing the awarded research in two of the main conferences of the field (see Table 1).

Table 1 - Papers (references and titles) analyzed in our survey.

\begin{tabular}{|l|l|}
\hline $\begin{array}{l}\text { [Hoffman and Weinberg, } \\
2010]\end{array}$ & $\begin{array}{l}\text { Gesture-based human-robot jazz improvi- } \\
\text { sation }\end{array}$ \\
\hline [Grollman and Billard, 2011] & $\begin{array}{l}\text { Donut as I do: Learning from failed } \\
\text { demonstrations }\end{array}$ \\
\hline [Bergstrom et al., 2011] & $\begin{array}{l}\text { Generating object hypotheses in natural } \\
\text { scenes through human-robot interaction }\end{array}$ \\
\hline [Thobbi et al., 2011] & Using human motion estimation for human- \\
\hline
\end{tabular}




\begin{tabular}{|l|l|}
\hline & robot cooperative manipulation \\
\hline [Tenorth et al., 2012] & $\begin{array}{l}\text { The RoboEarth language: Representing } \\
\text { and exchanging knowledge about actions, } \\
\text { objects, and environments }\end{array}$ \\
\hline [Daniel et al., 2012] & $\begin{array}{l}\text { Learning concurrent motor skills in versa- } \\
\text { tile solution spaces }\end{array}$ \\
\hline [Chu et al., 2013] & $\begin{array}{l}\text { Using robotic exploratory procedures to } \\
\text { learn the meaning of haptic adjectives }\end{array}$ \\
\hline [Fasola and Mataric, 2013] & $\begin{array}{l}\text { Using semantic fields to model dynamic } \\
\text { spatial relations in a robot architecture for } \\
\text { natural language instruction of service ro- } \\
\text { bots }\end{array}$ \\
\hline [Deisenroth et al., 2014] & Multi-task policy search for robotics \\
\hline [Gemici and Saxena, 2014] & $\begin{array}{l}\text { Learning haptic representation for manipu- } \\
\text { lating deformable food objects }\end{array}$ \\
\hline [Boularias et al., 2015] & $\begin{array}{l}\text { Grounding spatial relations for outdoor } \\
\text { robot navigation }\end{array}$ \\
\hline
\end{tabular}

As discussed in the previous section, in [Tedre, 2015] some classes of experiments are identified from the analysis of current practice in computing. According to our sample of representative papers, examples of experiments that fall in these classes are also largely present in autonomous robotics, although some new characterizations of experiments as explorations also emerge.

Feasibility experiments. These experiments are basically a form of empirical demonstration, intended as an existence of proof of the ability to build a robot system to perform some task. The outcome of a feasibility experiment is typically binary: positive, if the robot is able to accomplish what it is intended to do; negative, otherwise. Examples of this kind of experiments in the papers we analyzed are reported in Table 2 .

Table 2 - Some excerpts relative to feasibility experiments, according to the taxonomy proposed by [Tedre, 2015].

\begin{tabular}{|l|l|}
\hline [Tenorth et al., 2012] & $\begin{array}{l}\text { "The experiment shows that the system is } \\
\text { able to encode the information required for } \\
\text { mobile pick-and-place tasks" (p. 1289) }\end{array}$ \\
\hline [Fasola and Mataric, 2013] & $\begin{array}{l}\text { "These examples illustrate the ability of the } \\
\text { system to parse natural language input, } \\
\text { ground noun phrases, infer command } \\
\text { semantics, plan, and execute an appropriate } \\
\text { solution while obeying natural language } \\
\text { directive constraints" (p. 147) }\end{array}$ \\
\hline
\end{tabular}


Trial and field experiments. These experiments take a step further and evaluate various aspects of robot systems using some predefined variables which are measured in laboratories or in real contexts of use (with some limitations), in the case of trial experiments, or outside the laboratory in complex socio-technical contexts of use, in the case of field experiments. In these experiments, some quantities, like velocity and acceleration of parts of the robots, accuracy and time required for performing a task, or error with respect to a reference (ground truth), are measured to evaluate robot systems. Sometimes, measuring quantities amounts to resort to human judgement about the observed behavior of the robots. Some examples extracted from the papers we analyzed are reported in Table 3.

Table 3 - Some excerpts relative to trial and field experiments, according to the taxonomy proposed by [Tedre, 2015].

\begin{tabular}{|l|l|}
\hline [Grollman and Billard, 2011] & $\begin{array}{l}\text { "To evaluate our techniques we are } \\
\text { concerned not only with whether the task is } \\
\text { eventually performed successfully (which it } \\
\text { is), but also with the breadth of possibilities } \\
\text { that are generated" (p. 3807) }\end{array}$ \\
\hline [Thobbi et al., 2011] & $\begin{array}{l}\text { "Ten trials were performed to test how } \\
\text { quickly the algorithm could converge to an } \\
\text { optimal policy" (p. 2876) } \\
\text { "True velocity and acceleration are derived } \\
\text { from the observed position, and are shown } \\
\text { in the figure for comparison with the } \\
\text { predicted values" (p. 2876-2877) }\end{array}$ \\
\hline [Fasola and Mataric, 2013] & $\begin{array}{l}\text { "To evaluate the ability of our robot system } \\
\text { to follow natural language directives, we } \\
\text { first analyzed the effectiveness of the } \\
\text { semantic interpretation module to infer the } \\
\text { correct command specifications given the } \\
\text { natural language input" (p. 147) }\end{array}$ \\
\hline [Boularias et al., 2015] & $\begin{array}{l}\text { "Participants were separately asked to point } \\
\text { to the goal they would choose for executing } \\
\text { each command. The best answer, chosen by } \\
\text { a majority vote, is compared to the robot's } \\
\text { answer" (p. 1981) }\end{array}$ \\
\hline
\end{tabular}

Comparison experiments. These experiments refer to comparing different solutions in some set-ups and are based on some precisely-defined measures and criteria to assess the performance. The compared entities could be different versions of the robot system under testing (for instance, the same robot with or without a specific component) or alternative systems to perform the same task (for instance, systems proposed by other researchers). Table 4 shows some examples of this kind of experiments that are reported in the papers we analyzed. 
Table 4 - Some excerpts relative to comparison experiments, according to the taxonomy proposed by [Tedre, 2015].

\begin{tabular}{|l|l|}
\hline [Grollman and Billard, 2011] & $\begin{array}{l}\text { "Because there are more possibilities to } \\
\text { explore, in our experiments the donut } \\
\text { method took more interactions to succeed } \\
\text { than the balanced mean" (p. 3808) }\end{array}$ \\
\hline [Bergstrom et al., 2011] & $\begin{array}{l}\text { "Again, we conclude that point initialization } \\
\text { outperforms cluster initialization" (p. 832) } \\
\text { "In addition we evaluate how the method in } \\
\text { [10] compares to our method" (p. 833) }\end{array}$ \\
\hline [Thobbi et al., 2011] & $\begin{array}{l}\text { "Fig. 8 shows the trajectories of both ends } \\
\text { of the table for cases where the proposed } \\
\text { system was used (case I: with predictions) } \\
\text { and the case where only the reactive } \\
\text { controller was used (case II: without } \\
\text { predictions)" (p. 2877) }\end{array}$ \\
\hline [Daniel et al., 2012] & $\begin{array}{l}\text { "We also compare our approach to the } \\
\text { standard unimodal REPS algorithm" (p. } \\
\text { 3595) }\end{array}$ \\
\hline [Gemici and Saxena, 2014] & $\begin{array}{l}\text { "We compare the performance of our } \\
\text { reward based manipulation approach against } \\
\text { the baseline algorithms" (p. 644) }\end{array}$ \\
\hline
\end{tabular}

Controlled experiments. These experiments are the golden standard of experimentation in the natural sciences that refers to the original idea of experiment as controlled experience, where the activity of rigorously controlling (by adopting experimental principles such as reproducibility or repeatability) the factors that are under investigation is central, while eliminating the confounding factors, and allowing for generalization and prediction. In the current experimental practice of autonomous robotics (discussed at the beginning of this paper and in [Amigoni et al., 2014]), it is hard to find experimental activities that completely fit within this category.

Along with the above categories of experiments, the analyzed papers report evidence of other forms of empirical investigation on the functioning of artifacts and on their interaction with the environment, which are in the direction of explorative experiments and can be roughly organized in the following way according to their purposes $^{3}$. (Note that the categories below are separated for presentation clarity, but their boundaries are rather fuzzy.)

${ }^{3}$ Although it is out of the scope of the present paper to investigate the exact positioning of explorative experiments, we believe they represent an orthogonal dimension with respect to the five categories of experiments introduced by [Tedre, 2015] and discussed before. 
Investigating the role of parameters. Complex software programs controlling robot systems often involve several parameters whose values influence their behavior. For example, the software programs controlling robots could make decisions according to thresholds, or sensor data could be filtered according to factors depending on environment conditions. Often, the designer has only a rough a priori idea of the relationship between values of parameters and behavior of robot systems, and experiments are used to elucidate and make more precise this relationship. In a sense, the design of a robot system $\mathrm{R}$ requires tests in an experimental setting $\mathrm{S}$ in order to be refined with the proper values of parameters $\mathrm{P}$ that are good for S. To this end, some experiments reported in the surveyed papers are set up to elucidate the qualitative and quantitative effects of different parameters values on some measurable quantities relative to the behavior and the performance of the robots (Table 5).

Table 5 - Some excerpts relative to experiments devoted to investigate the role of parameters in robot systems.

\begin{tabular}{|l|l|}
\hline $\begin{array}{l}\text { [Hoffman and Weinberg, } \\
\text { 2010] }\end{array}$ & $\begin{array}{l}\text { "We have empirically sampled sound } \\
\text { intensity profiles for different solenoid } \\
\text { activation lengths, and used those to build a } \\
\text { model for each striker" (p. 583) }\end{array}$ \\
\hline [Thobbi et al., 2011] & $\begin{array}{l}\text { "Fig. 7 shows the role of the forgetting } \\
\text { factor } \phi \text { in determining the confidence" (p. } \\
\text { 2877) }\end{array}$ \\
\hline [Daniel et al., 2012] & $\begin{array}{l}\text { "We evaluate our approach with different } \\
\text { bounding parameters } k \text { for the } \\
\text { responsibilities. [...] In a second } \\
\text { experiment, we evaluate the influence of } \\
\text { importance sampling" (p. 3595) }\end{array}$ \\
\hline [Gemici and Saxena, 2014] & $\begin{array}{l}\text { "In this work, we manually tuned the reward } \\
\text { functions for our manipulation task for a } \\
\text { reasonable level of exploration and } \\
\text { exploitation" (p. 644) }\end{array}$ \\
\hline
\end{tabular}

Confirmation of expectations or hypotheses. When developing robot systems, the designers consider (and build upon) a set of expectations and hypotheses about the behavior of the artifacts when inserted in their operating environments. Usually, in autonomous robotics, due to the difficulty of building reliable models of the interaction between robots and the portion of the physical world in which they are inserted [Amigoni and Schiaffonati, 2010; Amigoni and Schiaffonati, 2014], these expectations and hypotheses are not based on a solid theoretical ground and can be confirmed only empirically. Schematically, the designer expects a robot system $\mathrm{R}$ to show behavior $\mathrm{B}$ when it is inserted in experimental setting $\mathrm{S}$, and would like to confirm such expectation. This class of experiments (Ta- 
ble 6 reports a sample taken from surveyed papers) provides a very simple feedback to the design phase in the context of a continuous iteration between design and experiments ${ }^{4}$. For example, they are used to provide a posteriori justifications on some assumptions, in the sense that the design of the robot system $\mathrm{R}$ is based on some hypotheses that are considered valid if $\mathrm{R}$ shows the expected behavior $\mathrm{B}$.

Table 6 - Some excerpts relative to experiments devoted to confirm expectations or hypotheses.

\begin{tabular}{|l|l|}
\hline [Grollman and Billard, 2011] & $\begin{array}{l}\text { "Further, as expected, exploration with both } \\
\text { techniques increased in the middle portions } \\
\text { of both tasks" (p. 3808) }\end{array}$ \\
\hline [Chu et al., 2013] & $\begin{array}{l}\text { "We first analyzed the feature vectors to } \\
\text { confirm that they capture meaningful } \\
\text { differences in the feel of the objects" (p. } \\
\text { 3053) } \\
\text { "This relatively low score supports our } \\
\text { belief that multiple motions should be } \\
\text { combined to increase the recognition of } \\
\text { haptic object properties" (p. 3054) } \\
\text { "which supports the hypothesis that our } \\
\text { methods can produce a meaningful set of } \\
\text { adjectives for completely new objects when } \\
\text { using all EPs" (p. 3054) }\end{array}$ \\
\hline
\end{tabular}

Getting insights on the behavior of the robot systems. In the most interesting cases, explorative experiments are used to get intuitions on how robot systems work and on how they perform tasks. Usually, these experiments provide quantitative results that the designers use to inspect, and possibly modify, the design of the internal methods of robot systems (see Table 7 for some examples taken from the surveyed papers). In this case, the qualitative or quantitative influence (or effect, or role) of module $\mathrm{M}$ of robot system $\mathrm{R}$ on behavior $\mathrm{B}$ (observed when $\mathrm{R}$ is put in an experimental setting S) are investigated. Note that experiments used with this explorative intention provide a richer knowledge than when used to investigate the role of parameters, ranging from measuring values of internal variables to generating ideas for alternative design solutions.

Table 7 - Some excerpts relative to experiments devoted to get insights on the behavior of robot systems.

\begin{tabular}{|l|l|}
\hline [Grollman and Billard, 2011] & $\begin{array}{l}\text { "We believe the decreased agreement at the } \\
\text { end of the movement comes from } \\
\text { accumulated drift during trajectory } \\
\text { generation" (p. 3808) }\end{array}$ \\
\hline
\end{tabular}

\footnotetext{
${ }^{4}$ Note that, in most of the papers we analyzed in our survey, this iteration process is only hinted and only final sucessful tests are described in detail.
} 


\begin{tabular}{|c|c|}
\hline & $\begin{array}{l}\text { "We believe this behavior (and some of the } \\
\text { visual jagginess) arises from our use of } \\
\text { gradient ascent in the velocity generation } \\
\text { and our initialization" (p. 3808) }\end{array}$ \\
\hline [Bergstrom et al., 2011] & $\begin{array}{l}\text { "The slightly lower performance of the } \\
\text { latter indicates that it might be better to let } \\
\text { the segments evolve on their own, rather } \\
\text { than giving a large bias from the start and } \\
\text { having the risk of getting stuck in local } \\
\text { minima" (p. 832) }\end{array}$ \\
\hline [Thobbi et al., 2011] & $\begin{array}{l}\text { "Fig. } 5 \text { shows the variation confidence (C) } \\
\text { through the task" (p. 2877) } \\
\text { "the trajectory is much smoother when the } \\
\text { human is placing the table down as } \\
\text { compared to moving upwards [...] It can } \\
\text { also be speculated that sophisticated } \\
\text { velocity or torque controlled robots would } \\
\text { yield smoother motions and offer better } \\
\text { improvements in performance using the } \\
\text { proposed technique" (p. 2878) }\end{array}$ \\
\hline [Fasola and Mataric, 2013] & $\begin{array}{l}\text { "To illustrate the usefulness of the semantic } \\
\text { field model towards representing static and } \\
\text { dynamic spatial relation primitives for use } \\
\text { in path generation and classification, Fig. } 6 \\
\text { shows the progression of the at, along, away } \\
\text { from, and in semantic field values along the } \\
\text { execution paths generated for test runs \#1-4, } \\
\text { respectively" (p. 148) }\end{array}$ \\
\hline [Gemici and Saxena, 2014] & $\begin{array}{l}\text { "This means that for most of the objects, } \\
\text { one or two information gathering actions } \\
\text { was enough to determine the best task } \\
\text { oriented action to reach the subgoal" (p. } \\
644 \text { ) }\end{array}$ \\
\hline [Boularias et al., 2015] & $\begin{array}{l}\text { "We notice that complex commands help } \\
\text { finding the right goals because they are less } \\
\text { ambiguous than simple commands" (p. } \\
\text { 1981) }\end{array}$ \\
\hline
\end{tabular}

Assessing the generality of robot systems. The most sophisticate way of employing experiments with an explorative flavor is to gain knowledge about the behavior of robot systems in settings that are different of those considered in their design, in order to evaluate the generality of these systems. In this case, a robot system $\mathrm{R}$ that has been designed and developed to perform in settings $\mathrm{S}$ is experimentally tested in settings S' (different from S). Table 8 reports some examples taken from the analyzed set of papers. For instance, settings $S$ ' could involve 
noisy data (as opposite to error-free data assumed during development and preliminary experiments) or data that the robot $\mathrm{R}$ has never seen before. This last aspect is particularly relevant in the case of learning systems, like those proposed in [Deisenroth et al., 2014] and [Gemici and Saxena, 2014]. In other cases, S' could involve special situations (e.g., unexpected behaviors of the humans that are interacting with R) that are excluded from S.

Table 8 - Some excerpts relative to experiments devoted to assess generality of robot systems.

\begin{tabular}{|l|l|}
\hline [Thobbi et al., 2011] & $\begin{array}{l}\text { "Fig. 6 shows a non-typical case where the } \\
\text { human chooses to take a pause during the } \\
\text { task" (p. 2877) }\end{array}$ \\
\hline [Fasola and Mataric, 2013] & $\begin{array}{l}\text { "To demonstrate the generalizability of our } \\
\text { approach and its usefulness in practice with } \\
\text { real robots in real environments, next we } \\
\text { present evaluation results of our robot } \\
\text { software architecture using maps of real } \\
\text { environments that were generated by } \\
\text { physical robots implementing SLAM with } \\
\text { onboard laser sensors" (p. 148) }\end{array}$ \\
\hline [Deisenroth et al., 2014] & $\begin{array}{l}\text { "We show that our MTPS approach allows } \\
\text { to generalize from demonstrated behavior to } \\
\text { behaviors that have not been observed } \\
\text { before" (p. 3880) }\end{array}$ \\
\hline [Gemici and Saxena, 2014] & $\begin{array}{l}\text { "In order to test the generalization of our } \\
\text { algorithm to new object categories, we also } \\
\text { included a new category (tofu) not seen } \\
\text { during training" (p. 645) }\end{array}$ \\
\hline
\end{tabular}

\section{Discussion}

The examples of the previous section show that if, on the one side, the attempt of autonomous robotics to conform its experimental methodology to that of controlled experiments is not yet fully (and perhaps cannot be) carried out, on the other side the current practice is characterized at various levels by a form of experimentation that seems to deal with exploration. A purely controlled form of experimentation is hardly possible due to the lack of some of the features that in the traditional protocol allow to control the experimental factors. In particular, in the case of autonomous robotics, modeling and predicting the behavior of the robot systems in their interaction with complex environments is not only far beyond 
the current and near-future technical knowledge, but it is also rather out of experimenter's control due to some intrinsic reasons: the experimenter is part of the system and (s)he is not in full control of the experimental set-ups [Kroes, 2015]. When we turn, instead, to the idea of exploration, experiments are seen as ways to explore possibilities, to investigate opportunities, and to give back information that is iteratively used to improve the artifacts both in their architecture and in their interaction with complex environments. What is explored is only partially known in advance, and surely not at the level of being expressed in the form of clear hypotheses derived from a strong theory to be tested later in (controlled) experimental campaigns. In a sense, explorative experiments are used to increase the confidence of designers on the behavior of their robot systems in physical environments.

In the context of the widened framework suggested in this paper, we attempt now a still primitive but - in our opinion - promising definition of explorative experiments that is shaped on the analysis of experiments in autonomous robotics, but that could hopefully be extended to other forms of experimentation in computer engineering and, especially, in artificial intelligence. By explorative experiments in autonomous robotics we mean experiments that are driven by the desire of investigating the realm of possibilities pertaining to the functioning of a robot system and to its interaction with the environment in absence of a proper theory or theoretical background. More precisely, explorative experiments are a special kind of directly action-guiding experiments which possess the following features:

- They are devoted to testing artifacts, meant as artificial entities purportedly built by humans to fulfill a purpose and, therefore, having a technical function.

- Their focus is to iteratively refine the intervention, meant as the union of knowledge and action characterizing experimental practice, and their ultimate purpose is not to test a general theory, but to probe the possibility and limits of the intervention.

- They do not force a sharp distinction between designers and experimenters and, instead, the practitioners often become experimenters.

- The control of the experimental factors cannot be fully managed from the beginning, but is in part carried out after the artifact has been inserted into its environment.

The reason why we use the term 'explorative' instead of 'exploratory' is to mark our difference from the philosophical work focused on accounting the distinction between exploratory and theory-driven experiments and based on the ways in which experiments depend on theory. In our attempt to characterize explorative experiments we are interested, instead, in the appeal to complexity that has been stressed in the philosophical literature [Burian, 2007], where some systems are considered too complicated to be investigated by means of a theorydriven approach. This appeal to complexity certainly applies to biology, but we believe that there are good reasons to extend it to computer engineering as well, in particular when the subjects of the experimentation are not just the artifacts per se, but rather the ways in which these artifacts are able to interact with the surround- 
ing physical and social environment. The reference to complexity helps in defining one important aspect we wish to stress in our characterization of explorative experiments: the fact that there is not sufficient information (in most of the cases for the lack of a proper theoretical background and/or previous experience) to provide precise expectations of what investigators will find. Thus, explorative experimentation is a way to find patterns of activities from which scientists could generate novel hypotheses to improve artifacts and gain confidence in their behavior. In this sense, explorative experiments are forms of empirical investigation of novel and interesting ideas or techniques, without the rigorous constraints of typical experimental methodologies. The role of explorative experiments appears thus particularly important in autonomous robotics, because such robot systems are developed to operate in environments that are largely unpredictable and difficult to capture in models, with the consequence that the designers can hardly anticipate the possible outcomes.

\section{Conclusions}

In this work, we have substantiated the need of reconsidering the traditional notion of experiments within the field of autonomous robotics. The partial, but significant, survey we have presented shows that forms of experimentation as explorations are already performed in the practice of the field. To account for these activities, we have proposed the idea of explorative experiments, as forms of directly action-guiding experiments inspired by the different elements discussed at the beginning of the paper, in order to widen the current experimental framework.

We plan to further refine the definition of explorative experiments, in particular in the direction of considering different forms of control, with respect to those adopted in the classic experimental paradigm, that take place a posteriori, after an artifact has been inserted into its environment. Moreover, the feedback that explorative experiments can provide on design of autonomous robots will be investigated in more detail. Finally, the questions relative to the limited repeatability and reproducibility of explorative experiments, which could lead to over-optimistic interpretations of results, will be addressed.

\section{References}

[Amigoni and Schiaffonati, 2010] F. Amigoni and V. Schiaffonati, "Good experimental methodologies and simulation in autonomous mobile robotics", in L. Magnani, W. Carnielli, and C. Pizzi (eds.), Model-Based Reasoning in Science and Technology, Springer, 2010, p. 315 332.

[Amigoni and Schiaffonati, 2014] F. Amigoni and V. Schiaffonati, "Autonomous mobile robot as technical artifacts: A discussion of experimental issues", in L. Magnani (ed.), ModelBased Reasoning in Science and Technology, Springer, 2014, p. 527-542. 
[Amigoni et al., 2014] F. Amigoni, V. Schiaffonati, and M. Verdicchio, "Good experimental methodologies for autonomous robotics: From theory to practice", in F. Amigoni and V. Schiaffonati (eds.), Methods and Experimental Techniques in Computer Engineering, SpringerBriefs in Applied Sciences and Technology, Springer, 2014, p. 37-53.

[Bergstrom et al., 2011] N. Bergstrom, M. Bjorkman, and D. Kragic, "Generating object hypotheses in natural scenes through human-robot interaction", Proc. IROS, 2011, p. 827-833.

[Bonsignorio and del Pobil, 2015] F. Bonsignorio and A. del Pobil, "Special issue on replicable and measurable robotics research", IEEE Robotics and Automation Magazine, 22, 3, 2015, p. $32-154$.

[Bonsignorio et al., 2015] F. Bonsignorio, J. Hallam, and A. del Pobil, "Special interest group on good experimental methodologies", http://www.heronrobots.com/EuronGEMSig/gem-sigevents, last visited November 2015

[Boularias et al., 2015] A. Boularias, F. Duvallet, J. Oh, and A. Stentz, "Grounding spatial relations for outdoor robot navigation", Proc. ICRA, 2015, p. 1976-1982.

[Burian, 1997] R. M. Burian, "Exploratory experimentation and the role of histochemical techniques in the work of Jean Brachet, 1938-1952", History and Philosophy of the Life Sciences, 19, 1997, p. 27-45.

[Chu et al., 2013] V. Chu, I. McMahon, L. Riano, C. McDonald, Q. He, J. Martinez PerezTejada, M. Arrigo, N. Fitter, J. Nappo, T. Darrell, and K. Kuchenbecker, "Using robotic exploratory procedures to learn the meaning of haptic adjectives", Proc ICRA, 2013, p. 30483055.

[Daniel et al., 2012] C. Daniel, G. Neumann, and J. Peters, "Learning concurrent motor skills in versatile solution spaces", Proc. IROS, 2012, p. 3591-3597.

[Deisenroth et al., 2014] M. Deisenroth, P. Englert, J. Peters, D. Fox, "Multi-task policy search for robotics", Proc. ICRA, 2014, p. 3876-3881.

[Fasola and Mataric, 2013] J. Fasola and M. Mataric, "Using semantic fields to model dynamic spatial relations in a robot architecture for natural language instruction of service robots", Proc. IROS, 2013, p. 143-150.

[Franklin, 2005] L. Franklin, "Exploratory experiments", Philosophy of Science, 72, 2005, p. 888-899.

[Gemici and Saxena, 2014] M. Gemici and A. Saxena, "Learning haptic representation for manipulating deformable food objects", Proc. IROS, 2014, p. 638-645.

[Grollman and Billard, 2011] D. Grollman and A. Billard, "Donut as I do: Learning from failed demonstrations", Proc. ICRA, 2011, p. 3804-3809.

[Hacking, 1983] I. Hacking, Representing and Intervening. New York: Cambridge University Press, 1983

[Hansson, 2015] S. O. Hansson, "Experiments before science? - What science learned from technological experiments", in S. O. Hansson (ed.), The Role of Technology in Science: Philosophical Perspectives, Dordrecht: Springer, 2015, p. 81-110.

[Hoffman and Weinberg, 2010] G. Hoffman and G. Weinberg, "Gesture-based human-robot jazz improvisation", Proc. ICRA, 2010, p. 582-587.

[Kroes, 2015] P. Kroes, "Experiments on socio-technical systems: The problem of control", Science and Engineering Ethics Special Issue on Experiments, Ethics, and New Technologies, 2015, DOI: 10.1007/s11948-015-9634-4.

[Rawseeds, 2015] The Rawseeds Project, http://www.rawseeds.org/home/, last accessed November 2015.

[RoCKIn, 2015] Robot Competitions Kick Innovation In Cognitive Systems and Robotics (RoCKIn), http://rockinrobotchallenge.eu, last accessed November 2015.

[Steinle, 1997] F. Steinle, "Entering new fields: Exploratory uses of experimentation", Philosophy of Science, 64, 1997, p. S65-S67.

[Tedre, 2011] M. Tedre, "Computing as a science: A survey of computing viewpoints", Minds and Machines, 21, 2011, p. 361-387.

[Tedre, 2015] M. Tedre, The Science of Computing. Boca Raton: CRC Press, Taylor \& Francis Group, 2015. 
[Tenorth et al., 2012] M. Tenorth, A. Perzylo, R. Lafrenz, and M. Beetz, "The RoboEarth language: Representing and exchanging knowledge about actions, objects, and environments", Proc. ICRA, 2012, p. 1284-1289.

[Thobbi et al., 2011] A. Thobbi, Y. Gu, and W. Sheng, "Using human motion estimation for human-robot cooperative manipulation", Proc. IROS, 2011, p. 2873-2878.

[Vermaas et al., 2011] P. Vermaas, P. Kroes, I. van de Poel, M. Franssen, and W. Houkes, A Philosophy of Technology. From Technical Artifacts to Sociotechnical Systems, Morgan \& Claypool, 2011.

[Waters, 2007] C. K. Waters, "The nature and context of exploratory experimentation", History and Philosophy of the Life Sciences, 19, 2007, p. 275-284. 